\title{
Joint Discussion 5 Modelling the Milky Way in the Era of Gaia
}

\author{
James J. Binney, Editor
}

Department of Theoretical Physics, University of Oxford, UK email:binney@thphys.ox.ac.uk

\begin{abstract}
The body of photometric and astrometric data on stars in the Galaxy has been growing very fast in recent years (Hipparcos/Tycho, OGLE-3, 2-Mass, DENIS, UCAC2, SDSS, RAVE, Pan Starrs, Hermes, ...) and in two years ESA will launch the Gaia satellite, which will measure astrometric data of unprecedented precision for a billion stars. On account of our position within the Galaxy and the complex observational biases that are built into most catalogues, dynamical models of the Galaxy are a prerequisite full exploitation of these catalogues. On account of the enormous detail in which we can observe the Galaxy, models of great sophistication are required. Moreover, in addition to models we require algorithms for observing them with the same errors and biases as occur in real observational programs, and statistical algorithms for determining the extent to which a model is compatible with a given body of data.

JD5 reviewed the status of our knowledge of the Galaxy, the different ways in which we could model the Galaxy, and what will be required to extract our science goals from the data that will be on hand when the Gaia Catalogue becomes available.
\end{abstract}

Keywords. Galaxy: stellar content, Galaxy:evolution, Galaxy: dynamics

SOC Members: Luis A.C. Aguilar, Herwig B. Dejonghe, Kenneth C. Freeman, Ortwin Gerhard, Naoteru Gouda, Amina Helmi, Jacques R. D. Lépine, Alice C. Quillen, Annie C. R. Robin, Natalia Y. Sotnikova. 